\title{
Knockdown of microRNA-1323 restores sensitivity to radiation by suppression of PRKDC activity in radiation-resistant lung cancer cells
}

\author{
YONG LI $^{1 *}$, WEI HAN ${ }^{1 *}$, TING-TING NI ${ }^{1 *}$, LIANG LU $^{1}$, MIN HUANG $^{2}$, YU ZHANG $^{1}$, \\ HUI CAO ${ }^{1}$, HAN-QUN ZHANG ${ }^{1}$, WEN LUO ${ }^{1}$ and HANG LI ${ }^{1}$ \\ ${ }^{1}$ Department of Oncology, Guizhou Provincial People's Hospital, Guiyang, Guizhou 550002; \\ ${ }^{2}$ Department of Occupational and Environmental Health, School of Public Health, \\ Ningxia Medical University, Ningxia 750004, P.R. China
}

Received November 23, 2014; Accepted March 2, 2015

DOI: 10.3892/or.2015.3884

\begin{abstract}
Resistance to radiation is a major problem in cancer treatment. The mechanisms of radioresistance remain poorly understood; however, mounting evidence supports a role for microRNAs (miRNAs) in the modulation of key cellular pathways mediating the response to radiation. The present study aimed to identify specific miRNAs and their effect on radioresistant cells. The global miRNA profile of an established radioresistant lung cancer cell line and the corresponding control cells was determined. Differential expression of the miRNAs was confirmed by quantitative realtime PCR (qRT-PCR). The binding effect of identical novel miRNAs and target mRNAs was determined by luciferase assay. Lung cancer cells were transfected with miRNAspecific mimics or inhibitors. The DNA-dependent protein kinase (DNA-PKcs) protein level was tested by western blot analysis. Radiosensitivity of cancer cells was determined using colony formation assay. Among the differentially expressed miRNAs, 25 miRNAs were overexpressed while 18 were suppressed in the radioresistant cells, both basally and in response to radiation compared to their control. An miRNA signature miR-1323 exhibited a $>5$-fold increase in the radioresistant cells. miR-1323 was demonstrated to bind to PRKDC 3'UTR, which is involved in DNA repair. Ectopic expression of miR-1323 significantly increased the survival fraction of irradiated cancer cells. Inhibition of miR-1323 reversed the radioresistance of cancer cells and subsequently
\end{abstract}

Correspondence to: Professor Hang Li, Department of Oncology, Guizhou Provincial People's Hospital, Guiyang, Guizhou 550002, P.R. China

E-mail: lihang.sy@163.com

*Contributed equally

Key words: microarray, miRNA, radio resistance, lung cancer, DNA repair suppressed the expression of miR-1323-regulated DNA-PKcs protein. The present study indicated that miRNAs are involved in the radioresistance of human lung cancer cells. A possible mechanism for resistance to radiation was via enhanced DNA repair. The present study demonstrated a role for miR-1323 in modulating radioresistance and highlights the need for further study investigating the potential role of miR-1323 as both a predictive marker of response and a novel therapeutic agent with which to enhance the efficacy of radiotherapy.

\section{Introduction}

Radiotherapy is the most commonly used therapeutic approach for managing cancer and acts mainly through the induction of DNA damage (1). Impairment in the DNA repair proteins, which physiologically protect cells from persistent DNA injury, can affect the efficacy of cancer therapy (2). The DNA damage repair (DDR) response is an intricate signal transduction pathway activated upon DNA damage. Cancer cells often show significant alterations at the level of the DDR response and develop resistance to DNA damage-inducing agents $(3,4)$.

Recently, increasing evidence suggests that microRNAs (miRNAs) take an active part in the regulation of the DNA damage/repair network $(5,6)$. miRNAs are endogenous short non-coding molecules able to regulate gene expression at the post-transcriptional level (7). In the last decade, miRNAs, a new class of molecules able to post-transcriptionally regulate gene expression, have emerged to be involved in several fundamental physiological and pathological biomolecular and cellular mechanisms $(8,9)$. miRNA profiles have been analyzed and several differentially expressed miRNAs involved in many cellular functions such as apoptosis, cell cycle control, and DNA damage/repair were identified in response to different radiation doses (10).

miRNAs are non-encoding small RNAs existing extensively in plants, animals and viruses, at an approximate length of 21-23 nt and are highly conserved. They bind to a specific mRNA 3'UTR and regulate gene transcription. Mature miRNAs together with other proteins form into RNA-induced silencing complex, resulting in the degradation or translation 
suppression of target mRNAs when binding to a target mRNA 3'UTR (11).

The analysis of miRNA-modulated gene regulation in the DDR and its involvement in cancer pathogenesis and progression will help to understand and define the impact of these small molecules in DNA damage/repair as well as chemoresistant and radioresistant mechanisms $(12,13)$. This knowledge will expand the characterization of molecules and networks involved in pathways activated upon DNA damage and the subsequent alterations at the level of fundamental processes such as cell cycle control and apoptosis (14). The identification of miRNA-modulated genes and the effects of deregulated functions of miRNAs will make it possible to acquire information concerning the prognosis, chemoresistance or radioresistance, and then the response to therapeutic treatments in cancer. However, the implications of miRNAmRNA interactions in genotoxic mechanisms remain unknown (15).

In the present study, we investigated the global miRNA expression in an established radioresistant lung cancer cell line from our previous study and analyzed the identified miRNA-mRNA interaction to radiotherapy. The aim of this study was to clarify the mechanisms of resistance in lung cancer cells and to also identified specific miRNAs in lung cancer cells following radiation treatment.

\section{Materials and methods}

Cells and cell culture. The human lung cancer cell line A549 was purchased from the American Type Culture Collection (ATCC) and cultured in RPMI-1640 with Dulbecco's modified Eagle's medium (Sigma-Aldrich, St. Louis, MO, USA) supplemented with $20 \%$ fetal calf serum, $0.05 \%$ L-glutamine, $150 \mathrm{UI} / \mathrm{ml}$ penicillin and $50 \mu \mathrm{g} / \mathrm{ml}$ streptomycin in a humidified atmosphere with $5 \% \mathrm{CO}_{2}$ at $37^{\circ} \mathrm{C}$. The cultures were split every second day by dilution at a concentration of $2 \times 10^{5}$ cells $/ \mathrm{ml}$. The cell counts were performed with a hemocytometer; the cell membrane integrity was determined using the trypan blue exclusion technique. Cell lines in the maximal range of up to 20 passages were used for the present study. All cell lines tested negative for mycoplasma contamination by polymer chain reaction (PCR) methods (16). Cell lines were authenticated using the short tandem repeats (STR) testing.

Development of acquired radioresistant cells. A549 cells $\left(1 \times 10^{6}\right)$ were plated in $75-\mathrm{cm}^{2}$ culture flasks and irradiated with 4 Gy of $\gamma$-rays using a Theratron Cobalt-60 treatment unit (Siemens, Concord, CA, USA) at a dose rate of $1 \mathrm{~Gy} / \mathrm{min}$ when the cells were at $\sim 60 \%$ confluency in the culture flask. Immediately following irradiation, the culture medium was renewed, and the cells were returned to the incubator. When the A549 cells reached $\sim 90 \%$ confluency, they were trypsinized, counted and passaged into new culture flasks. Again, the cells were treated with 4-Gy $\gamma$-rays when they reached $\sim 60 \%$ confluency. The irradiation was performed 13 times for a total dose of 60 Gy (irradiated with 2 Gy of $\gamma$-rays at the final irradiation) over 5 months. The parental cells were trypsinized, counted and passaged under the same conditions without irradiation.
In vitro $X$-ray irradiation. The final cell density was adjusted to $1 \times 10^{6}$ cells $/ \mathrm{ml}$, and the samples were placed at $37^{\circ} \mathrm{C}$ in a $5 \%$ $\mathrm{CO}_{2}$ incubator. X-irradiation was performed using a Theratron Cobalt-60 treatment unit (Siemens) at a dose rate of $2 \mathrm{~Gy} / \mathrm{min}$. Non-irradiated cells were treated in similar way, but at a zero radiation dose.

Colony formation assay. The cells were trypsinized and resuspended in T25 flasks, followed by $\gamma$-ray exposure at room temperature at single doses of $0,4,8$, and 12 Gy. After irradiation, the cells were seeded onto $6-\mathrm{cm}$ dishes and incubated for 14 days without disturbance. The seeded cell number was increased as the radiation dose increased. Formed colonies were visualized by staining with $0.02 \%$ crystal violet solution (w/v in $75 \%$ ethanol). The plating efficiency was determined as the ratio of the number of colonies divided by the number of cells seeded. The surviving fraction was determined from the ratio of plating efficiencies of irradiated cells compared to the no irradiated control. Each data value represents the mean of three independent experiments \pm SEM.

Total RNA preparation. The samples were ground by using liquid nitrogen. Total RNA was extracted from lung tissues using an animal tissue RNA purification kit (Norgen Biotek, Thorold, ON, USA) as recommended by the manufacturer. RNA samples were measured using Bioanalyzer to determine RNA integrity number.

miRNA microarray chip analysis. miRNA expression profiling was performed using the TaqMan Rodent miRNA Array Card A and Card B (Applied Biosystems, Foster City, CA, USA). Each chip contained multiple quality control probes and employed dual-color chip to examine miRNA expression profiling in mouse lung tissues. Probes were synthesized in situ with photosensitive PGR. The sequence consisted of 2 fragments, namely, a chemically modified oligonucleotide encoding fragment complementary to target miRNA or other target RNA, and an extension arm at a certain distance to the connected encoding sequence, which lessened the hybridization spatial impairment. The temper of probe hybridization was balanced by the chemical modification. Cy3- and Cy5-specific fluorescent labels and Axon GenePix 4000B microarray scanner were used to capture hybridization images. ArrayPro (Media Cybernetics, Bethesda, MD, USA) was utilized to complete the digital transformation.

Quantitative real-time PCR ( $q R T-P C R)$. An approximate length of 21-23 nt of miRNA resulted in difficulties in conventional PCR test. We used TaqMan ${ }^{\circledR}$ microRNA assays (Applied Biosystems) to examine the miRNA differential expression profiling in PTC as recommended by the manufacturer. Sample RNA (10 ng) was reversely transcribed into cDNA by using specific stem-loop primers and TaqMan ${ }^{\circledR}$ microRNA reverse transcription kit. With cDNA as the template, TaqMan microRNA assay and TaqMan ${ }^{\circledR}$ Universal PCR Master Mix were used for the serial real-time PCR. RNU48 was used as an internal control to minimize the variation among reverse transcription, PCR and samples. Data were collected, analyzed, and normalized using the Applied Biosystems analysis software to determine the differential expression profiles of the 

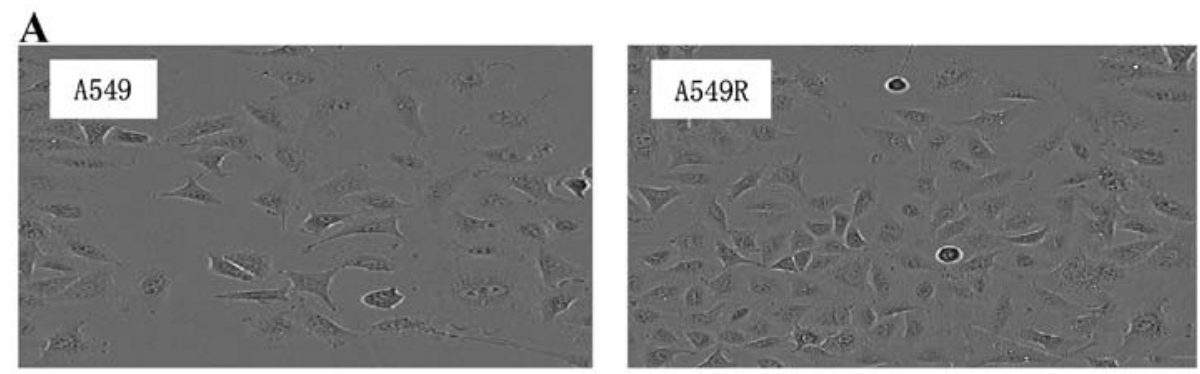

B

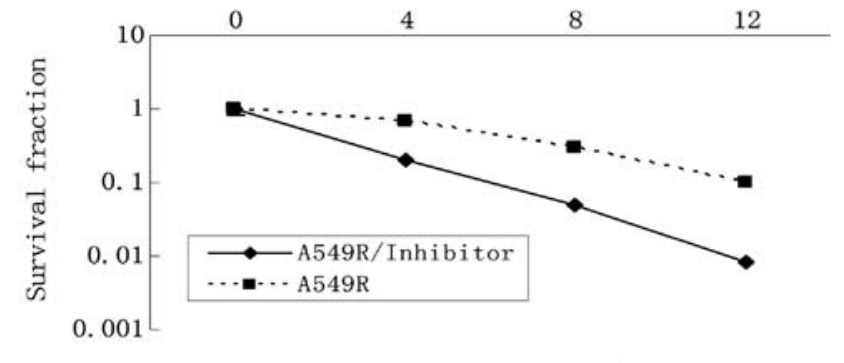

Radiation dose (Gy)

Figure 1. Morphology and radiosensitivity of the A549 cells. (A) No obvious change in cell morphology was observed following radiation. (B) The radioresistant A549R cells had a higher survival rate than the non-radioresistant A549 cells.

miRNAs. All of the experiments were conducted in triplicate. Expression levels were calculated using the relative quantification method $(\Delta \Delta C T)$ in the ABI PRISM 7500 sequence detection system (Applied Biosystems), according to the manufacturer's instructions.

miRNA overexpression and knockdown. miR-1323 was overexpressed by reverse transfection of $100 \mathrm{nM}$ pre-miR precursor molecule using siPORT NeoFX transfection reagent as per the manufacturer's instructions and compared with the pre-miR negative control (miR-control) (all from Life Technologies/Ambion). Inhibition of miR-1323 was achieved by reverse transfection of $30 \mathrm{nM}$ anti-miR-1323 inhibitor (Life Technologies/Ambion) using siPORT NeoFX transfection reagent and compared with the anti-miR negative control.

Luciferase constructs and assays. Full-length human PRKDC 3'UTR sequences were amplified by PCR and cloned into the Spe1/HindIII restriction sites of the pMIR Report Luciferase plasmid (Life Technologies/Invitrogen, Carlsbad, CA, USA). The putative miR-1323 target sites in the 3'UTR of PRKDC were mutated using the QuikChange XL Site-Directed Mutagenesis kit (Stratagene, La Jolla, CA, USA). Resistant cells were transfected with $750 \mathrm{ng}$ wild-type or mutant PRKDC 3'UTR luciferase reporter constructs and stable cell lines generated by puromycin selection. Stable cell lines were reverse transfected with $10 \mathrm{nM}$ miR-1323 or miR-control. Cells were incubated for a further $24 \mathrm{~h}$, and luciferase activity was assessed using the Dual-Luciferase assay system (Promega, Fitchburg, WI, USA).

Western blot analysis. Proteins were resolved on $12 \%$ polyacrylamide gels, transferred to a nitrocellulose membrane (Bio-Rad, Hercules, CA, USA) and blocked with 5\% non-fat dairy milk in Tris-buffered saline $(20 \mathrm{mM}$ Tris and $150 \mathrm{mM} \mathrm{NaCl}, \mathrm{pH} 7.4$ ) with $0.1 \%$ Tween-20.
Statistical and bioinformatic analysis of the microarray data. In the process and analysis of data, the background was initially excluded. Any 'bad spot' that showed a signaling value deviation above $50 \%$ of the average of repeated spots and/or a spot CV above 0.5 was deleted preceding the calculation of the mean and standard deviation for repeated spots. The normalization was completed by locally weighted regression. For the double-labeling experiments, the ratio of 2 sets of detected signals ( $\log 2$ transformed) and P-value for the Student-t test were calculated. A P-value $<0.05$ was considered to be statistically significant. Significantly differential miRNAs were defined as those showing a $\operatorname{logarithm}(\log 2)$ for the ratio of the treatment group signal to control signal $\geq 1$ or $\leq-1$. Multiple target gene prediction software including miRanda (http://www.mocrorna.org), TargetScan (http://www.targetscan.org), mirTarget2 (http:// mirdb.org/miRDB), RNA22 (http://cbcsrv.watson.ibm.com/ rna22.html), and microTv3.0 (http://diana.cslab.ece.ntua.gr/ microT) were used to forecast some potential target genes showing a sequential differential expression profiling. The genes identified by at least 3 software, were taken as target genes to minimize the false positivity. In order to further characterize the molecular functions of forecasted target genes, MAS database (http://bioinfo.capitalbio.com/mas3/) and GDM database (http://gdm.fmrp.usp.br/) were used to make a preliminary analysis of forecasted target genes.

\section{Results}

Morphology and radiosensitivity of the A549R cells. The radioresistant cells designated as A549R, were obtained by subjecting A549 cells to 5 months of fractionated irradiation with a total dose of 60 Gy and 10 additional passages without irradiation. No obvious change in the cell morphology was observed following irradiation (Fig. 1A). The radiosensitivity of the A549 and A549R cells was compared using a colony 
A

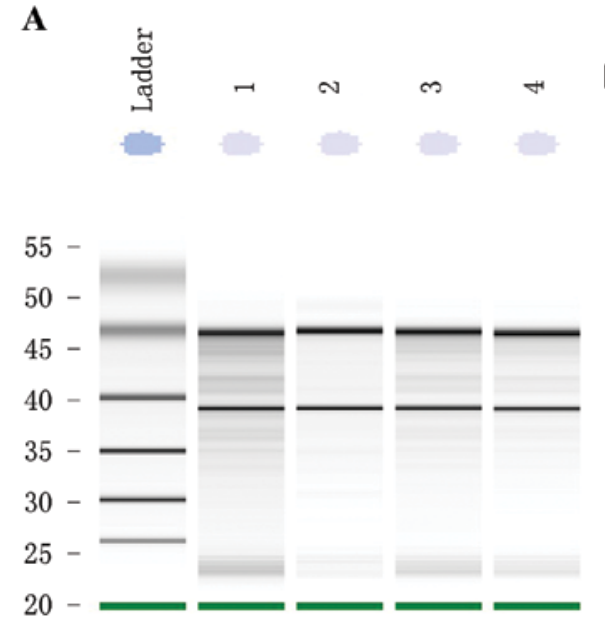

B

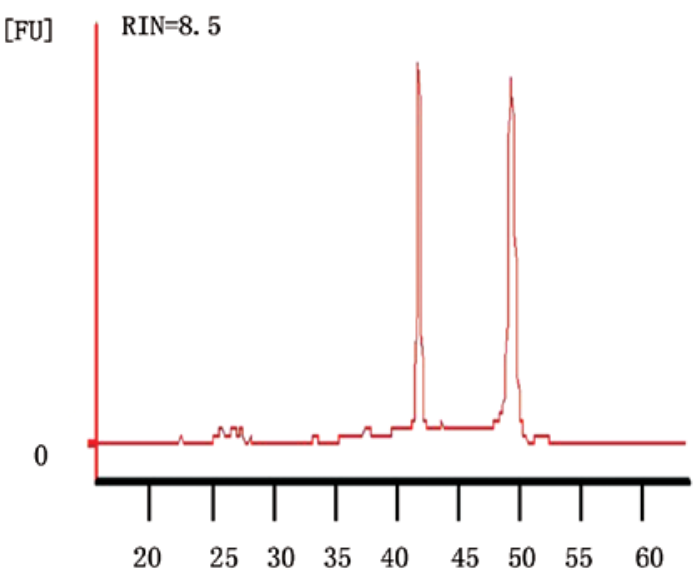

Figure 2. Determining the integrity of RNA. (A) A total RNA sample showing a detailed image of the size distribution of RNA fragments on the Agilent 2100 Bioanalyzer using the Eukaryote Total RNA Nano assay. (B) Representative electropherograms for RIN classes.

Table I. Differential expression of miRNAs in the resistant subclone and parental cells (resistance/parental).

\begin{tabular}{|c|c|c|c|c|c|c|c|}
\hline No. & miRNA & Fold change $^{\mathrm{a}}(\log 2)$ & $\mathrm{P}$-value & No. & miRNA & Fold change $(\log 2)$ & P-value \\
\hline 1 & miR-1323 & 13.234 & 0.0032 & 23 & miR-375 & 2.344 & 0.0008 \\
\hline 2 & $\operatorname{miR}-34 c$ & 11.422 & 0.0051 & 24 & $\operatorname{miR}-744$ & 2.123 & 0.0013 \\
\hline 3 & miR-181 & 11.233 & 0.0178 & 25 & miR-140-3p & 2.098 & 0.0340 \\
\hline 4 & $\operatorname{miR}-214$ & 9.966 & 0.0020 & 26 & miR-142-5p & -14.098 & 0.0043 \\
\hline 5 & miR-31 & 8.434 & 0.0411 & 27 & miR-122 & -9.856 & 0.0237 \\
\hline 6 & miR-148a & 8.234 & 0.0069 & 28 & miR-7 & -9.678 & 0.0198 \\
\hline 7 & miR-299-5p & 8.012 & 0.0260 & 29 & miR-146a & -7.345 & 0.0260 \\
\hline 8 & $\operatorname{miR}-127$ & 6.987 & 0.0145 & 30 & $\operatorname{miR}-126$ & -5.345 & 0.0103 \\
\hline 9 & miR-660 & 6.782 & 0.0071 & 31 & $\operatorname{miR}-424$ & -5.231 & 0.0071 \\
\hline 10 & miR-22 & 6.340 & 0.0124 & 32 & miR-494 & -5.013 & 0.0342 \\
\hline 11 & miR-24-1 & 5.233 & 0.0048 & 33 & miR-224 & -4.875 & 0.0304 \\
\hline 12 & miR-766 & 5.123 & 0.0021 & 34 & miR-155 & -4.456 & 0.0102 \\
\hline 13 & miR-762 & 5.076 & 0.0161 & 35 & miR-638 & -4.123 & 0.0051 \\
\hline 14 & miR-143 & 4.671 & 0.0052 & 36 & miR-483-3p & -4.091 & 0.0178 \\
\hline 15 & miR-376a & 4.234 & 0.0225 & 37 & miR-92a & -3.987 & 0.0003 \\
\hline 16 & miR-99a & 4.123 & 0.0146 & 38 & miR-505 & -3.675 & 0.0311 \\
\hline 17 & miR-132 & 4.098 & 0.0301 & 39 & miR-125-3p & -3.102 & 0.0254 \\
\hline 18 & miR-145 & 3.567 & 0.0169 & 40 & miR-572 & -2.514 & 0.0260 \\
\hline 19 & $\mathrm{miR}-125 \mathrm{~b}$ & 3.123 & 0.0024 & 41 & miR-100 & -2.287 & 0.0175 \\
\hline 20 & miR-130a & 2.987 & 0.0071 & 42 & $\operatorname{miR}-720$ & -2.173 & 0.0091 \\
\hline 21 & miR-423-5p & 2.871 & 0.0176 & 43 & let-7e & -2.098 & 0.0105 \\
\hline 22 & $\operatorname{miR}-497$ & 2.456 & 0.0248 & & & & \\
\hline
\end{tabular}

${ }^{a}$ Fold changel $>2$ is listed; fold change equals resistance/parental.

formation assay (Fig. 1B). Each point on the survival curve represents the mean surviving fraction from triplicate experiments. As expected, the A549R cells had a higher survival rate than the A549 cells, indicating that the A549R cells were more radioresistant than the $\mathrm{A} 549$ cells.

Purity and integrity of RNA. RNA extracted from the parental and resistant cells showed an RIN number $>8$, suggesting the high purity of total RNA without any protein or DNA residual (Fig. 2). The formaldehyde denaturing agarose gel electrophoresis image showed that the 28S:18S band neared 2:1 in a clear and trailing-free manner, without any non-specific band, suggesting the integrity of degradation-free RNA. The quality control experiment confirmed the suitability of the RNA sample for further miRNA microarray and reverse transcription PCR. 


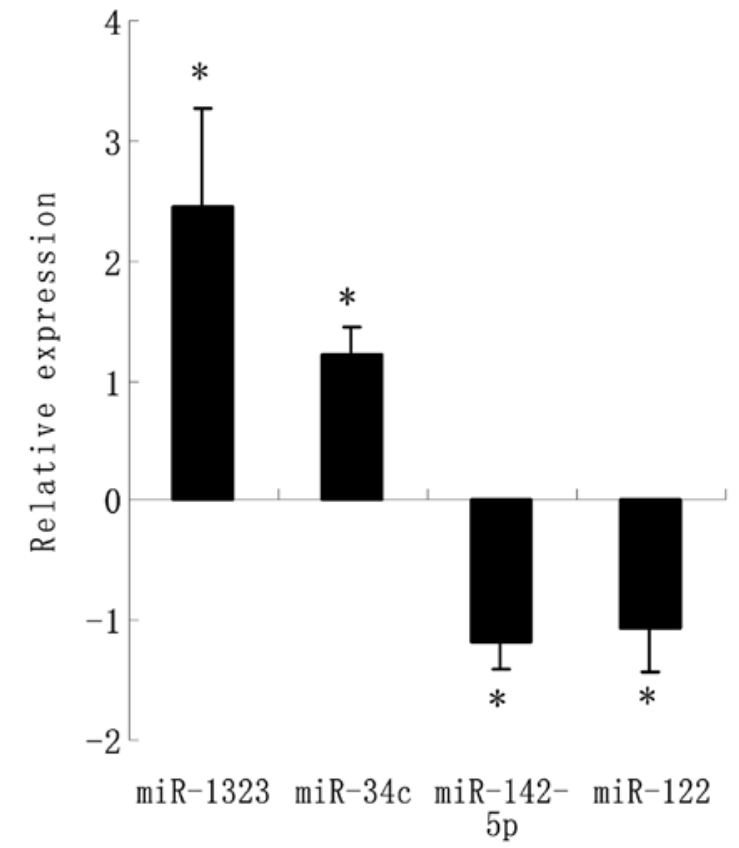

Figure 3. Real-time quantitative PCR (RT-qPCR) validation between resistance and parental control. RT-qPCR validation of microRNA array results. Data are presented as average fold change over matched controls (unexposed) (mean \pm SEM). Expression values were normalized to small nuclear RNA RNU1A1. Significant differences by the Student's t-test ( $\mathrm{P}<0.05)$.

Expression of miRNAs and $q R T-P C R$ verification between the control and acquired radioresistant cells. A microarray platform optimized for the analysis of a panel of human miRNAs was used to analyze and compare the pattern of miRNA expression between the parental A549 cell line and its counterpart resistant cell line. The expression profile of 43 miRNAs significantly changed (2.0- to 14.0 -fold) including 25 upregulated miRNAs and 18 downregulated miRNAs in the resistant cells as compared to the parental cells (Table I). The four most significantly changed miRNA were chosen to be verified by RT-PCR which showed the same results as the array analysis (Fig. 3). Among these, miR-1323 had the highest fold-change in the exposure tissues. Compared to those in the parental cells, miR-1323 was significantly upregulated and miR-142-5p was significantly downregulated $(\mathrm{P}<0.05)$, respectively, in the resistance exposure group.

miR-1323 directly suppresses expression of the luciferase reporter containing the PRKDC $3^{\prime} U T R$. To identify miRNAs that regulate human PRKDC expression, we used the web-based algorithms TargetScan, miRanda and PITA. All these tools predicted a putative binding site for miR-1323 in the PRKDC 3'UTR (Fig. 4A). To validate the interaction between miR-1323 and the PRKDC 3'UTR, we cloned full-length wild-type PRKDC 3'UTR into the reporter vector to serve as the $3^{\prime}$ UTR of the luciferase coding region. Acquired resistant cells were used to generate stable wild-type PRKDC-luciferase cell lines. These cells were then transfected with miR-1323 or miR-control, as a negative control, and luciferase activity was measured. miR-1323 overexpression increased luciferase activity driven by PRKDC 3'UTRs. To determine whether this effect is direct, the predicted miR-1323-binding sites
$\mathbf{A}$

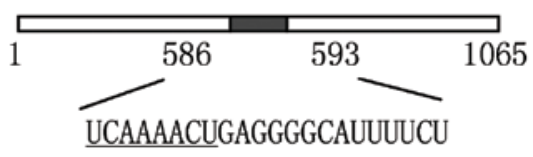

B

miR-1323: 3' UCUUUUACGGGGAGUCAAAACU 5'

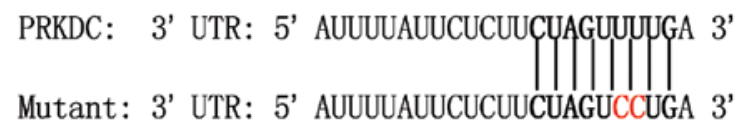

C

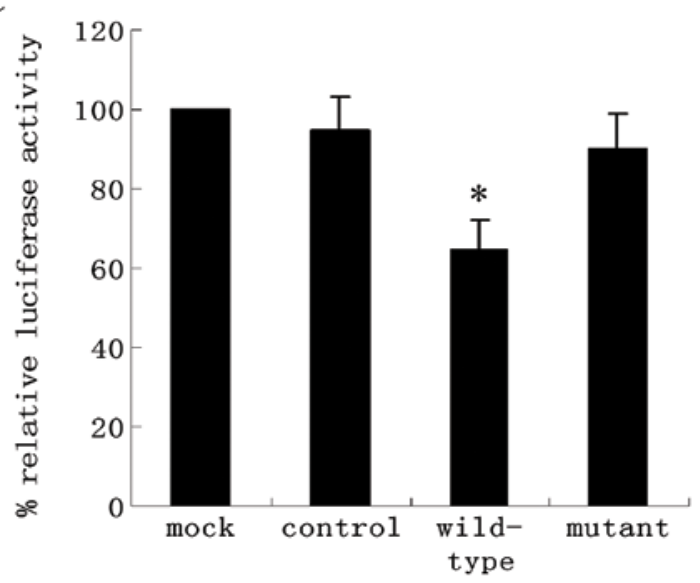

Figure 4. Identification of an miR-1323 target site and effect of the putative binding site from the 3'UTR of PRKDC mRNA on luciferase expression. (A) Schematic of the PRKDC 3'UTR (\#NM_001081640) nucleotides 586-593 showing the predicted miR-1323 binding site in the 3'UTR. The actual nucleotide sequences in PRKDC mRNA and miR-1323 show a partial match. (B) Alignment of miR-1323 with the insert derived from the PRKDC 3'UTR. Note the complementarity at the 5' end of miR-1323, where the crucial seed region is located. A single-based mutant insert was also synthesized, as shown. Inserts were cloned into the 3'UTR of the p-miR-report vector. (C) Luciferase activity in cells transiently transfected with the luciferase construct alone, or co-transfected with an expression plasmid for miR-1323. Luciferase vectors included control, luciferase with the PRKDC-derived 3'UTR insert, or luciferase with the mutated insert (mutant $3^{\prime} \mathrm{UTR}$ ). ${ }^{*} \mathrm{P}<0.05$, vs. control for relative luciferase activity.

in the $3^{\prime} \mathrm{UTR}$ of luciferase-PRKDC plasmids were mutated generating luciferase PRKDC-mutant constructs (Fig. 4B). Co-transfection of lung cells with the parental luciferase construct without the PRKDC 3'UTR did not significantly change the expression of the reporter. However, when the miR-1323 target site from the PRKDC 3'UTR was inserted into the luciferase construct, expression of luciferase was strongly increased when co-transfected with miR-1323 (Fig. 4C). This enhanced expression was suppressed by a single-base mutation in the binding site. Taken together, these data indicate that miR-1323 interacts with specific elements in the 3'UTR of PRKDC.

miR-1323 promotes the production of PRKDC. To investigate whether miR-1323 modulates endogenous PRKDC expression, we examined the effects of miR-1323 overexpression on PRKDC protein levels in human primary lung cell lines. Upon transfection, miR-1323 was overexpressed 5-fold in the resistant cells as determined by real-time quantitative PCR 

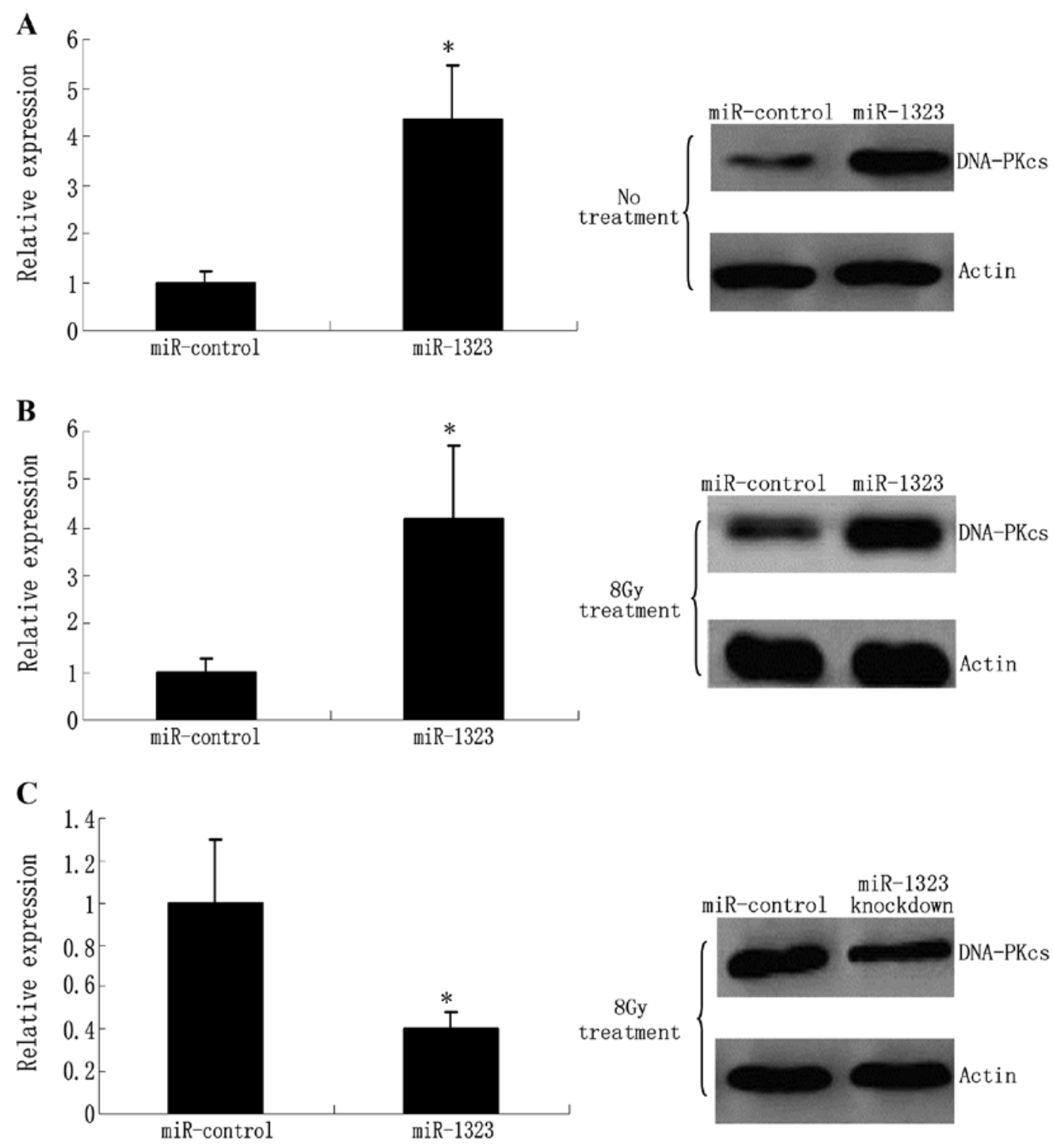

Figure 5. miR-1323 is inversely correlated with DNA-PKcs levels in response to radiation. (A) Resistant cells were transfected with miR-1323, and miR-1323 levels were determined by real-time quantitative PCR (RT-qPCR). Western blot analysis was performed to determine DNA-dependent protein kinase (DNAPKcs) protein levels. (B) Resistant cells were treated with 8-Gy radiation. miR-1323 and the DNA-PKcs protein level were determined. (C) miR-control and miR-1323 knockdown cells were treated with 8-Gy radiation. miR-1323 and the DNA-PKcs protein level were determined. Actin served as the loading control, ${ }^{*} \mathrm{P}<0.05$.

(RT-qPCR) (Fig. 5A). Nevertheless, miR-1323 overexpression caused an increase in the DNA-PKcs protein levels compared with the miR-control in the cell lines (Fig. 5A).

miR-1323 knockdown effects cellular response to radiation. Owing to the role of DNA-dependent protein kinases (DNA-PKcs) in response to genotoxic insults, we explored the regulation of miR-1323 and DNA-PKcs following genotoxic stress stimuli. Firstly, resistant cells were treated with 8-Gy radiation. Radiation-induced accumulation of DNA-PKcs protein was found to correlate with a concomitant increase in miR-1323 levels (Fig. 5B). These data suggest that an increase in miR-1323 and accumulation of DNA-PKcs are a general response to DNA double-break DNA adducts caused by radiation. To understand the role of miR-1323 in response to DNA damage, we knocked down miR-1323 in resistant cells that were then treated with $8 \mathrm{~Gy}$. Unlike the control cells,
miR-1323-knockdown cells failed to recruit the DNA-PKcs protein (Fig. 5C).

\section{Discussion}

In an effort to better understand the mechanisms underlying radioresistance, different radioresistant cell models, including the glioma MGR2R (17), glioblastoma U251 (18), colon adenocarcinoma WiDr (19) and small cell lung cancer cell line H69 (20), have been generated using distinct IR exposure methods. Repeated low-dose IR exposure and sublethal IR exposure are the most frequently adopted strategies that are used to establish radioresistant cancer cell models. In our opinion, the gradually increasing doses of IR exposure decreased the potential for cellular contamination after repeated low-dose IR exposure and avoided sudden cell death during sublethal IR exposure. However, the manner in which 
IR exposure can maximally enhance the radioresistant capability remains to be investigated in the future (10).

miRNAs are considered to be involved in multiple malignant cell behaviors including radioresistance $(21,22)$. Previous studies have used miRNA arrays to identify different miRNA expression profiles that are associated with cancer initiation and progression (23-25). In the present study, high-throughput sequencing technology was applied to discover novel miRNAs associated with radioresistance. Our results demonstrated that 18 known miRNAs were downregulated and 25 known miRNAs were upregulated. Some of the previously identified miRNAs have been reported to regulate the proliferation, apoptosis, migration and invasion of cancer cells and have prognostic value (10).

Among these, miR-1323 exhibited the highest fold-change in the radioresistant cells. Further functional study revealed its binding site on PRKDC (NM_001081640). This gene encodes the catalytic subunit of the DNA-PK. It functions with the $\mathrm{Ku} 70 / \mathrm{Ku} 80$ heterodimer protein in DNA doublestrand break repair and recombination. The protein encoded is a member of the PI3/PI4 kinase family. miR-1323 is located on chromosome 19. This sequence was identified as an miRNA candidate by Berezikov et al (26), and was confirmed later by cloning (27). A previous study reported that it was overexpressed in radioresistant NPC cancer cells and it had significantly lower expression in skeletal muscle from aged persons (28). All of these implied its possible critical role in the DNA repair pathway.

Studies have identified some novel miRNAs which are involved in the DNA repair pathway and regulate the DNA damage response to radiation (29-31). Yan et al (32) identified miR-101 as a molecule able to sensitize cancer cells to IR by targeting the 3'UTR of DNA-PKcs and ATM transcripts. The authors demonstrated that miR-101 overexpression could be used for rendering tumor cells more sensitive to radiation in in vitro and in vivo models. miR-18a was able to affect DNA damage response mechanisms through ATM downregulation (33). miR-18a was overexpressed in breast cancer cell lines and tumors and its ectopic expression downregulated ATM by direct interaction with the 3'UTR of the gene. ATM siRNA and miR-18a overexpression caused reduction of homologous recombination and DNA repair in breast cancer cells, making them more sensitive to ionizing radiation. Conversely, inhibition of miR-18a led to an increase in homologous recombination and DNA repair efficiency, thus reducing cellular radiosensitivity. Several miRNAs able to inhibit $\gamma \mathrm{H}_{2} \mathrm{AX}$ foci formation were identified. miR-138 specifically targeted the $\mathrm{H}_{2} \mathrm{AX}$ 3'UTR, reducing its expression and inducing chromosomal instability after DNA damage. miR-138 overexpression inhibited homologous recombination and increased sensitivity to DNA damaging agents. Chang et al (34) showed that miR-3928 was induced by ionizing radiation in HeLa cells and targeted the endoribonuclease Dicer. miR-3928 overexpression promoted ATR activation and Chk1 phosphorylation. miR-3928 overexpression was also able to downregulate several miRNAs, including miR-185, miR-300, and miR-663. Additionally, Wang et al (35) demonstrated that miR-185, whose expression is reduced after ionizing radiation exposure in renal cell carcinoma ( $\mathrm{RCC}$ ), targeted ATR. miR-185 expression sensitized RCC cells to X-rays both in vivo and in vitro and enhanced radiation-induced apoptosis as well as inhibition of proliferation by repressing the ATR pathway. Therefore, miR-185 could be potentially used to radiosensitize cancer cells.

In conclusion, miRNAs involved in the regulation of DNA damage/repair mechanisms can be considered as markers to predict the response to radiotherapy and can be utilized hereafter to define personalized treatments (36). The identification of miRNAs that are associated with radioresistance may lead to more individualized and efficient treatments for cancer patients. In this regard, expression levels of miRNAs could be evaluated in serum and/or tumor specimens to predict radiosensitivity and optimal radiation dose, in order to make the treatment more effective and to limit both side effects and normal tissue injury. In addition, expression and activity of miRNAs able to affect the response to chemotherapy or radiotherapy could be specifically modified and modulated to enhance the expected therapeutic effects. The use of artificial miRNAs with sequences able to target genes already known to have important roles in DNA damage/ repair mechanisms could be of great impact in regulating mechanisms able to render cancer cells more sensitive to DNA damaging agents.

\section{Acknowledgements}

We acknowledge the Guizhou Province Programs for Science and Technology Development.

\section{References}

1. Begg AC, Stewart FA and Vens C: Strategies to improve radiotherapy with targeted drugs. Nat Rev Cancer 11: 239-253, 2011.

2. Kastan MB: DNA damage responses: mechanisms and roles in human disease: 2007 G.H.A. Clowes Memorial Award Lecture. Mol Cancer Res 6: 517-524, 2008.

3. Hosoya $\mathrm{N}$ and Miyagawa K: Targeting DNA damage response in cancer therapy. Cancer Sci 105: 370-388, 2014 (Epub ahead of print).

4. Lomax ME, Folkes LK and O'Neill P: Biological consequences of radiation-induced DNA damage: relevance to radiotherapy. Clin Oncol (R Coll Radiol) 25: 578-585, 2013.

5. Czochor JR and Glazer PM: microRNAs in cancer cell response to ionizing radiation. Antioxid Redox Signal 21: 293-312, 2014 (Epub ahead of print).

6. Metheetrairut $\mathrm{C}$ and Slack FJ: MicroRNAs in the ionizing radiation response and in radiotherapy. Curr Opin Genet Dev 23: 12-19, 2013.

7. Meltzer PS: Cancer genomics: Small RNAs with big impacts. Nature 435: 745-746, 2005.

8. Trang P, Weidhaas JB and Slack FJ: MicroRNAs as potential cancer therapeutics. Oncogene 27 (Suppl 2): S52-S57, 2008.

9. Farazi TA, Spitzer JI, Morozov P and Tuschl T: miRNAs in human cancer. J Pathol 223: 102-115, 2011.

10. Li G, Qiu Y, Su Z, Ren S, Liu C, Tian Y and Liu Y: Genome-wide analyses of radioresistance-associated miRNA expression profile in nasopharyngeal carcinoma using next generation deep sequencing. PLoS One 8: e84486, 2013.

11. Bartel DP: MicroRNAs: target recognition and regulatory functions. Cell 136: 215-233, 2009.

12. Weidhaas JB, Babar I, Nallur SM, Trang P, Roush S, Boehm M, Gillespie E and Slack FJ: MicroRNAs as potential agents to alter resistance to cytotoxic anticancer therapy. Cancer Res 67: 11111-11116, 2007.

13. Liu ZL, Wang H, Liu J and Wang ZX: MicroRNA-21 (miR-21) expression promotes growth, metastasis, and chemo- or radioresistance in non-small cell lung cancer cells by targeting PTEN. Mol Cell Biochem 372: 35-45, 2013.

14. Wouters MD, van Gent DC, Hoeijmakers JH and Pothof J: MicroRNAs, the DNA damage response and cancer. Mutat Res 717: 54-66, 2011. 
15. Tessitore A, Cicciarelli G, Del Vecchio F, Gaggiano A, Verzella D, Fischietti M, Vecchiotti D, Capece D, Zazzeroni F and Alesse E: MicroRNAs in the DNA damage/repair network and cancer. Int J Genomics 2014: 820248, 2014.

16. Uphoff CC and Drexler HG: Detecting mycoplasma contamination in cell cultures by polymerase chain reaction. Methods Mol Biol 731: 93-103, 2011.

17. Cheng JJ, Hu Z, Xia YF and Chen ZP: Radioresistant subline of human glioma cell line MGR2R induced by repeated high dose X-ray irradiation. Ai Zheng 25: 45-50, 2006 (In Chinese).

18. Lee HC, Kim DW, Jung KY, Park IC, Park MJ, Kim MS Woo SH, Rhee CH, Yoo H, Lee SH, et al: Increased expression of antioxidant enzymes in radioresistant variant from U251 human glioblastoma cell line. Int J Mol Med 13: 883-887, 2004.

19. Virsik-Köpp P, Hofman-Hüther H, Rave-Fränk $M$ and Schmidberger $\mathrm{H}$ : The effect of wortmannin on radiation-induced chromosome aberration formation in the radioresistant tumor cell line WiDr. Radiat Res 164: 148-156, 2005.

20. Henness S, Davey MW, Harvie RM and Davey RA: Fractionated irradiation of H69 small-cell lung cancer cells causes stable radiation and drug resistance with increased MRP1, MRP2, and topoisomerase IIalpha expression. Int J Radiat Oncol Biol Phys 54: 895-902, 2002.

21. Su H, Jin X, Zhang X, Xue S, Deng X, Shen L, Fang Y and Xie C: Identification of microRNAs involved in the radioresistance of esophageal cancer cells. Cell Biol Int 38: 318-325, 2014.

22. Wang P, Zhang J, Zhang L, Zhu Z, Fan J, Chen L, Zhuang L, Luo J, Chen H, Liu L, et al: MicroRNA 23b regulates autophagy associated with radioresistance of pancreatic cancer cells Gastroenterology 145: 1133-1143, e12, 2013.

23. Wang XC, Wang W, Zhang ZB, Zhao J, Tan XG and Luo JC: Overexpression of miRNA-21 promotes radiation-resistance of non-small cell lung cancer. Radiat Oncol 8: 146, 2013.

24. Besse A, Sana J, Fadrus P and Slaby O: MicroRNAs involved in chemo- and radioresistance of high-grade gliomas. Tumour Biol 34: 1969-1978, 2013

25. Lin J, Liu C, Gao F, Mitchel RE, Zhao L, Yang Y, Lei J and Cai J: miR-200c enhances radiosensitivity of human breast cancer cells. J Cell Biochem 114: 606-615, 2013.

26. Berezikov E, van Tetering G, Verheul M, van de Belt $J$, van Laake L, Vos J, Verloop R, van de Wetering M, Guryev V, Takada S, et al: Many novel mammalian microRNA candidates identified by extensive cloning and RAKE analysis. Genome Res 16: 1289-1298, 2006.
27. Afanasyeva EA, Hotz-Wagenblatt A, Glatting $\mathrm{KH}$ and Westermann F: New miRNAs cloned from neuroblastoma. BMC Genomics 9: 52, 2008.

28. Mercken EM, Majounie E, Ding J, Guo R, Kim J, Bernier M, Mattison J, Cookson MR, Gorospe M, de Cabo R, et al: Age-associated miRNA alterations in skeletal muscle from rhesus monkeys reversed by caloric restriction. Aging (Albany, NY) 5: 692-703, 2013.

29. Gasparini P, Lovat F, Fassan M, Casadei L, Cascione L, Jacob NK, Carasi S, et al: Protective role of miR-155 in breast cancer through RAD51 targeting impairs homologous recombination after irradiation. Proc Natl Acad Sci USA 111: 4536-4541, 2014 (Epub ahead of print)

30. Guo P, Lan J, Ge J, Nie Q, Guo L, Qiu Y and Mao Q: miR-26a enhances the radiosensitivity of glioblastoma multiforme cells through targeting of ataxia-telangiectasia mutated. Exp Cell Res 320: 200-208, 2014.

31. Liu YJ, Lin YF, Chen YF, Luo EC, Sher YP, Tsai MH, Chuang EY and Lai LC: MicroRNA-449a enhances radiosensitivity in CL1-0 lung adenocarcinoma cells. PLoS One 8: e62383, 2013.

32. Yan D, Ng WL, Zhang X, Wang P, Zhang Z, Mo YY, Mao $\mathrm{H}$ Hao C, Olson JJ, Curran WJ, et al: Targeting DNA-PKcs and ATM with miR-101 sensitizes tumors to radiation. PLoS One 5: e11397, 2010.

33. Song L, Lin C, Wu Z, Gong H, Zeng Y, Wu J, Li M and Li J: miR-18a impairs DNA damage response through downregulation of ataxia telangiectasia mutated (ATM) kinase. PLoS One 6: e25454, 2011.

34. Chang L, Hu W, Ye C, Yao B, Song L, Wu X, Ding N, Wang J and Zhou G: miR-3928 activates ATR pathway by targeting Dicer. RNA Biol 9: 1247-1254, 2012.

35. Wang J, He J, Su F, Ding N, Hu W, Yao B, Wang W and Zhou G: Repression of ATR pathway by miR-185 enhances radiation-induced apoptosis and proliferation inhibition. Cell Death Dis 4: e699, 2013

36. Zhao L, Bode AM, Cao Y and Dong Z: Regulatory mechanisms and clinical perspectives of miRNA in tumor radiosensitivity. Carcinogenesis 33: 2220-2227, 2012. 